\title{
S Research Square \\ Moving From Evidence To Decisions in Guidelines: An Analysis of Guidance Documents
}

\section{Jose F Meneses-Echavez ( $\nabla$ jose.meneses@fhi.no)}

Kunnskapssenteret for helsetjenesten i Folkehelseinstituttet https://orcid.org/0000-0003-4312-6909

\section{Julia Bidonde}

Norwegian Institute of Public Health Knowledge Centre for the Health Services: Folkehelseinstituttet

Formidling og bruk av kunnskap

\section{Juan Jose Yepes-Nuñez}

Universidad de Los Andes Facultad de Medicina

\section{Tina Poklepović Peričić}

University of Split Faculty of Medicine: Sveuciliste u Splitu Medicinski fakultet

\section{Livia Puljak}

Catholic University of Croatia: Hrvatsko katolicko sveuciliste

\section{Malgorzata M Bala}

Jagiellonian University Medical College Faculty of Medicine: Uniwersytet Jagiellonski Collegium Medicum Wydzial Lekarski

\section{Dawid Storman}

Jagiellonian University Medical College Faculty of Medicine: Uniwersytet Jagiellonski Collegium Medicum Wydzial Lekarski

\section{Mateusz Swierz}

Jagiellonian University Medical College Faculty of Medicine: Uniwersytet Jagiellonski Collegium Medicum Wydzial Lekarski

\section{Joanna Zając}

Jagiellonian University Medical College Faculty of Medicine: Uniwersytet Jagiellonski Collegium Medicum Wydzial Lekarski

\section{Camila Montesinos-Guevara}

UTE University Faculty of Health Sciences: Universidad UTE Facultad de Ciencias de la Salud Eugenio Espejo

\section{Yuan Zhang}

McMaster University Department of Health Research Methods Evidence and Impact

\section{Nathaly Chavez Guapo}

Universidad de La Sabana

\section{Holger Schünemann}

McMaster University Department of Health Research Methods Evidence and Impact

\section{Signe Flottorp}


Norwegian Knowledge Centre for the Health Services: Folkehelseinstituttet Formidling og bruk av kunnskap

\section{Pablo Alonso-Coello}

CIBERESP: Centro de Investigacion Biomedica en Red de Epidemiologia y Salud Publica

\section{Research}

Keywords: Practice guidelines, Decision Making, Evidence-Based Practice, Methods

Posted Date: January 10th, 2022

DOI: https://doi.org/10.21203/rs.3.rs-1193859/v1

License: (c) (1) This work is licensed under a Creative Commons Attribution 4.0 International License. Read Full License 


\section{Abstract \\ Background}

The process of moving from evidence to decisions (EtD) represents a cornerstone within guideline development methods. Little is known about the processes used by organizations in charge of guideline development and what criteria they consider when formulating recommendations.

\section{Objective}

To identify and describe the processes suggested for the formulation of healthcare recommendations in health care guidelines available in guidance documents.

\section{Methods}

We searched in spring 2020 the Guidelines International Network (G-I-N) website, MEDLINE, and The Cochrane Methodology Register to retrieve guidance documents published in the last decade by organizations dedicated to guideline development. Pairs of researchers independently selected and extracted data about the characteristics of the guidance document, including explicit or implicit recommendation-related criteria and processes considered, as well as the use of frameworks. We conducted both descriptive and bivariate analyses.

\section{Results}

We included 68 guidance documents, published mostly by scientific societies (58\%). Most of the organizations reported a system for grading the strength of recommendations (88\%), half of them being the GRADE approach. Two out of three guidance documents (66\%) proposed the use of a framework to guide the EtD process. We identified 14 recommendation-related criteria. The GRADE Evidence to Decision (GRADE-EtD) framework was the most often reported framework (19 organizations, $42 \%$ ), whereas 20 organizations (44\%) proposed their own multi-criteria frameworks. Using any EtD framework was related with a more comprehensive set of recommendation-related criteria compared to no framework, especially for criteria like values, equity, and acceptability. A similar association was observed between the GRADEEtD framework and either no framework or another EtD frameworks.

\section{Conclusion}

The use of systematic and structured processes for moving from evidence to decisions is still limited among international organizations. The use of EtD frameworks facilitates the inclusion of relevant recommendation criteria. Among the structured frameworks, the GRADE-EtD framework offers the most 
comprehensive perspective for evidence-informed decision-making processes. More complete and detailed reporting in the guidance documents is warranted.

\section{Contributions To The Literature}

- The use of Evidence to Decision (EtD) frameworks facilitates the consideration of all important criteria for the recommendations when conducting clinical practice guidelines.

- The use of structured EtD frameworks is limited among international organizations devoted to guideline development.

- Using an EtD framework results in higher probabilities of considering a comprehensive set of recommendation-related criteria compared with no framework, as well as for the use of the GRADEEtD framework compared to another framework or no framework.

- Organizations should follow more systematic and explicit methods for formulating recommendations and ensure a complete reporting in their guidance documents.

\section{Background}

Guidelines for clinical, public health and health policy, hereafter guidelines, enhance decision-making by translating complex scientific research findings into recommendations for practice $(1 ; 2)$. Guideline developers share the same aim, establishing standards of care backed by strong scientific evidence; however, they do not share the same methodological expertise and resources for guideline development $(1 ; 2)$. According to the National Academy of Medicine, trustworthy guidelines should "be developed by a knowledgeable, multidisciplinary panel of experts and representatives from key affected groups; be based on a systematic review of the existing evidence; consider important patient subgroups and patient preferences as appropriate; be based on an explicit and transparent process that minimizes distortions, biases, and conflicts of interest; provide a clear explanation of the logical relationships between alternative care options and health outcomes and provide ratings of both the quality of evidence and the strength of the recommendations; and be reconsidered and revised as appropriate when important new evidence warrants modifications (2)".

The process of moving from evidence to recommendations represents a cornerstone of guideline development $(2 ; 3)$. This process implies a systematic and transparent integration of the evidence supporting the criteria influencing the recommendation, as expressed by item 13 in the Guidelines 2.0 checklist (3). Due to the broad variety of recommendation-related criteria suggested by international organizations (2-7), the McMaster checklist, a tool for guideline development, points out that a framework outlining the criteria to be considered to arrive at a decision (e.g., the magnitude of the difference between benefits and harms, and resource use) should guide the recommendation formulation process (3).

A number of frameworks have been proposed for addressing this process, such as the GRADE- Evidence to Decision (EtD) framework (4), the 'decision-making triangle' (8), the EVIDEM framework (6), or the 
Guidance for Priority Setting in Health Care (GPS-Health) (9). Some research groups and organizations have launched other EtD frameworks and processes, mostly based on the criteria and subcriteria contained in the GRADE-EtD framework (10;11). Some of EtD frameworks emphasize specific criteria, such as equity in the GPS-Health (9), or ethics as the 'decision-making triangle' (8). Despite the variation in terms of the criteria being proposed, all the EtD frameworks aim to offer a comprehensive list of criteria needed to be considered by both decision makers and guideline developers.

One of the most popular frameworks is the GRADE-EtD. There exists a growing body of research about the role of the GRADE-EtD framework in guideline development (12). The GRADE Working Group developed the EtD framework for different types of decisions, including recommendations (4). Details about the development process of the GRADE-EtD framework are available elsewhere (13). Briefly, the GRADE-EtD framework helps guideline development groups (GDG) to considering the most relevant criteria that influence a decision by structuring discussions, identifying reasons for disagreements, and building the basis for a transparent decision $(4 ; 13$ ) (see Figure 1). The GRADE-EtD framework has been used by international organizations including the World Health Organization (WHO), the European Commission (7) and major scientific societies (14-16). Some panelists have acknowledged the relevance of the framework in facilitating both structured panel meetings and the consideration of all relevant criteria pertaining decision making (17). However, the controversy around the best fit for purpose framework remains in the literature, explained by the fact that new proposals continue to emerge even in recent years (10).

Despite the progress achieved in studying the importance of various recommendation-related criteria, to our knowledge, the use of the EtD process in guidance documents has not been yet evaluated systematically. Therefore, we conducted a systematic identification and description of the processes suggested for the formulation of recommendations in health care guidelines available in guidance documents.

\section{Methods}

\section{Design}

Methodological study. We followed the methods for the conduct of a systematic review (e.g. double data extraction) (18). We published the protocol in Open Science Framework (19).

\section{Inclusion criteria}

We included methodological guidance documents for guideline development available as organization reports, journal articles, or briefs published by institutions responsible for guideline development. We discarded guidance documents dealing with processes of updating or adapting guidelines as well as documents endorsing included documents.

\section{Literature searches}


We systematically searched the Guidelines International Network (G-I-N) library, MEDLINE, Google Scholar, and The Cochrane Methodology Register using key terms such as "handbook*", "guideline methodology", "clinical practice guideline*", and "manual". Then, we inspected the references listed in the included documents and websites of relevant organizations identified in previous research projects $(1 ; 20-22)$, with focus on the most recent version or edition of the guidance document. These procedures (search and inspection of references) were first conducted during the Fall 2018, and further updated in Spring 2020. The searches had no language restrictions except for Google Scholar, which was restricted to English. We contacted experts in the field. Additional file 1 describes the search strategies.

\section{Study selection and data extraction}

We designed and piloted an ad hoc data abstraction form based on the WHO handbook for guideline development (7) because of its comprehensiveness, relevance, and formative influence on other guideline documents. The form covered information on the main characteristics of the guidance documents, as well as characteristics of the methodology (e.g. panel composition and management of conflict of interests), and information on the EtD process, including the use of frameworks (4). We defined a structured process as any systematic series of steps taken in order to fulfill a goal, and a framework as any structure of concepts underlying a structured process, in this case the process of formulating recommendations (EtD process). We explored the full-text of each guidance document for information about the suggested EtD process, including the use of frameworks, and listed all recommendation-related criteria considered. Both selection and data extraction processes were undertaken independently by pairs of reviewers, with discrepancies solved by consensus or by involving a third reviewer, if needed.

\section{Data management and analysis}

Pairs of reviewers read the guidance documents and extracted the information pertaining the recommendation formulation process (e.g., direct quotes). We held rounds of virtual meetings to ensure accuracy and completeness of the data. All data extraction forms were then compiled by one reviewer (JFME), who run descriptive analysis, with frequencies and percentages (e.g., characteristics of the organizations, panel composition and conflicts of interest management). Based on the EtD process and the recommendation-related criteria identified in the guidance documents, we created the following categories:

1) Use of any framework for the EtD process

1.1.) Use of GRADE-EtD framework

1.2) Use of other EtD framework

2) No framework

The last step of the analysis involved the study of the probability of addressing each criterion across the different categories of frameworks presented above (i.e., any framework vs no framework; GRADE-EtD vs 
other EtD framework; GRADE-EtD vs no framework; other EtD framework vs no framework), for which we estimated Odds Ratios (OR) with their corresponding 95\% confidence intervals (Cl). We used R-project software for the analysis (23). JFME carried out the statistical analysis, and a second reviewer (PAC) audit it for accuracy.

\section{Results}

We included a total of 68 guidance documents from 14 countries. Additional file 2 presents the names of the organizations and their corresponding guidance documents included in this study. The median publication year of the documents was 2015 (range 2003-2020). Scientific societies published most of the documents (58\%), followed by governmental (20\%), and supranational organizations (13\%). Nearly half of the documents were from North America (45\%), followed by Europe (37\%), Asia and Oceania (6\%), and South America (3\%). See Figure 2 and Additional file 3 for further details on the characteristics of the included guidance documents. Figure 2 does not show six guidance documents published by European organizations, World Health Organization (WHO) and World Confederation for Physical Therapy (WCPT).

Most of the guidance documents provided some degree of information about the composition of guideline panels. A multidisciplinary panel, including a steering group, methodologists with expertise in evidence synthesis, and health professionals specialized in the topic of interest was the most common composition. More than half of the documents (64\%) suggested the involvement of patients or consumer representatives (Additional file 3). Half of the guidance documents presented a definition of conflicts of interest (COIs) and presented a process to report the COls. Although $87 \%$ of the guidance documents specified who should report COls, only half of them stated that any declaration of COls should be reviewed before making appointments to the GDG and specified the types of COls to be declared (i.e., financial and non-financial). Half of the documents (59\%) described a process for the management of COls. See Additional file 3.

\section{Panel composition and conflicts of interest management Rating the quality of evidence and grading the strength of recommendations}

Almost all guidance documents (93\%) suggested a structured approach or system for rating the quality of the evidence, most of them (85\%) contained a specific section or chapter. GRADE was the most common approach suggested (53\%), followed by the approaches proposed by the Australian National Health and Medical Research Council (NHMRC), and the Scottish Intercollegiate Guidelines Network (SIGN), reported in three documents each (4\%). Of note, seven documents $(10 \%)$ suggested rating systems that were based on GRADE or other approaches. Finally, nine documents (13\%) proposed their own systems, mainly based on the previous approaches. See Additional file 2 for further details. 
Most of the guidance documents (88\%) proposed a system for grading the strength of recommendations; half of them suggested the GRADE approach. Around a fifth of the documents (22\%) suggested their own approach, whereas $14 \%$ of the documents did not report any. Other approaches were SIGN (3\%), Oxford Centre for Evidence-Based Medicine (1 document), NICE (1 document), and USPSTF (1 document). Five documents (8\%) proposed other approaches, mostly adapted from GRADE (Table 1). 
Table 1

Recommendation formulation information reported in the included guidance documents

$\mathrm{N}(\%)$

Contains specific section in the document

$55(81 \%)$

Details of people involved

- Entire panel/GDG (8, $56 \%)$

- Panel + other $(5$, $7 \%)$

- Subgroup of the panel $(3,4 \%)$

- Not specified (19, $28 \%)$

Technical team shares materials (e.g., evidence summaries) with the guideline panel ahead of meeting

$27(40 \%)$

Technical team or someone else makes preliminary judgments on the different criteria (e.g., certainty of evidence).

$25(37 \%)$

Technical team or someone else makes preliminary judgment on the strength of recommendations (e.g., strong, conditional).

$15(22 \%)$

Technical team or someone else makes preliminary judgments about the direction of recommendations (e.g., in favor against).

$7(10 \%)$

Approach to grading the strength of recommendations

$60(88 \%)$

AHRQ: The Agency for Healthcare Research and Quality (USA); CEBM: The Centre for Evidence-Based Medicine, based in the Nuffield Department of Primary Care Health Sciences at the University of Oxford; GDG: Guideline development group; GRADE: The Grading of Recommendations Assessment, Development and Evaluation approach; NHMRC: National Health and Medical Research Council (Australia); NICE: The National Institute for Health for Care and Excellence (UK); SIGN: Scottish Intercollegiate Guidelines Network; USPSTF: The U.S. Preventive Services Task Force 


\begin{tabular}{|c|c|}
\hline & $N(\%)$ \\
\hline \multirow[t]{12}{*}{ Approach suggested for grading the strength of recommendations } & - GRADE $(35,51 \%)$ \\
\hline & $-\operatorname{NHMRC}(2,3 \%)$ \\
\hline & $-\operatorname{SIGN}(2,3 \%)$ \\
\hline & - CEBM $(1,1.5 \%)$ \\
\hline & - NICE $(1,1.5 \%)$ \\
\hline & - USPSTF $(1,1.5 \%)$ \\
\hline & $\begin{array}{l}\text { - Adapted systems (5, } \\
7 \%)\end{array}$ \\
\hline & $\begin{array}{l}\text { GRADE + NICE (1, } \\
1.5 \%)\end{array}$ \\
\hline & NICE + SIGN $(2,3 \%)$ \\
\hline & $\begin{array}{l}\text { GRADE + SIGN (1, } \\
1.5 \%)\end{array}$ \\
\hline & $\begin{array}{l}\text { GRADE + AHRQ + } \\
\text { USPSTF }(1,1.5 \%)\end{array}$ \\
\hline & $\begin{array}{l}\text { - Other or not } \\
\text { specified } 21(36 \%)\end{array}$ \\
\hline Use of a framework for the EtD process & $45(66 \%)$ \\
\hline \multirow[t]{8}{*}{ Frameworks suggested for the EtD process } & GRADE-EtD: 19 (42\%) \\
\hline & $\begin{array}{l}\text { Other approaches: } 26 \\
(58 \%)\end{array}$ \\
\hline & $\begin{array}{l}\text { Own approach } 20 \\
(76 \%)\end{array}$ \\
\hline & NICE $2(8 \%)$ \\
\hline & SIGN $1(4 \%)$ \\
\hline & USPSTF $1(4 \%)$ \\
\hline & SIGN+NICE 1 (4\%) \\
\hline & $\begin{array}{l}\text { GRADE+SIGN+AHRQ } \\
1(4 \%)\end{array}$ \\
\hline
\end{tabular}

AHRQ: The Agency for Healthcare Research and Quality (USA); CEBM: The Centre for Evidence-Based Medicine, based in the Nuffield Department of Primary Care Health Sciences at the University of Oxford; GDG: Guideline development group; GRADE: The Grading of Recommendations Assessment, Development and Evaluation approach; NHMRC: National Health and Medical Research Council (Australia); NICE: The National Institute for Health for Care and Excellence (UK); SIGN: Scottish Intercollegiate Guidelines Network; USPSTF: The U.S. Preventive Services Task Force 


\section{$N(\%)$}

Explicit method to reach agreement among panel members (e.g., consensus, $\quad 56$ (82\%) nominal group techniques).

Documentation of judgements made.

$27(38 \%)$

AHRQ: The Agency for Healthcare Research and Quality (USA); CEBM: The Centre for Evidence-Based Medicine, based in the Nuffield Department of Primary Care Health Sciences at the University of Oxford; GDG: Guideline development group; GRADE: The Grading of Recommendations Assessment, Development and Evaluation approach; NHMRC: National Health and Medical Research Council (Australia); NICE: The National Institute for Health for Care and Excellence (UK); SIGN: Scottish Intercollegiate Guidelines Network; USPSTF: The U.S. Preventive Services Task Force

\section{Recommendation formulation}

Two out of three guidance documents (66\%) suggested a structured process for formulating recommendations, with the entire panel being involved in more than half $(56 \%)$ of the documents. In contrast, $28 \%$ of the guidance documents failed to report who was involved in the process of formulating recommendations. The technical team needed to share preliminary material (e.g., evidence summaries and evidence-to-recommendation tables) with the guideline panel ahead of GDG meetings in $40 \%$ of the guidance documents, and $48 \%$ of the documents reported that the technical team should make preliminary judgments about the quality of the evidence. A lower number of documents $(37 \%)$ declared that preliminary judgments on the certainty of the evidence or any other factor (e.g., values and preferences, equity, resources required) should be made before the panel meetings. A smaller number of documents $(22 \%)$ suggested the same for deciding about the direction and strength of recommendations (10\%) (see Table 1).

\section{Use of EtD frameworks}

The GRADE-EtD framework was the most often reported (42\%), followed by NICE's framework (8\%), SIGN (4\%), and USPSTF (4\%). Twenty guidance documents (76\%) reported their own multi-criteria framework. None of the guidance documents reported the use of multi-criteria decision analysis frameworks. Nearly half of the documents (57\%) provided guidance for formulating recommendations when there is insufficient evidence or no evidence available, or low/very low-quality evidence. Most of the documents $(82 \%)$ suggested a method to reach agreement among guideline panel members (e.g., consensus, voting (majority rule), or nominal group techniques) (see Table 1).

\section{Setting, perspective, and subgroup considerations}

We found that $42 \%$ and $24 \%$ of the guidance documents that included an EtD framework specified the setting and perspective, respectively. These rates were considerably lower among documents that did not suggest any framework (17\% setting and $9 \%$ perspective). Guidance documents suggesting the GRADEEtD framework were the most common ones in reporting the inclusion of both setting and perspective 
(42\%), followed by those that suggested another EtD framework ( $42 \%$ and $11 \%$, respectively). The inclusion of subgroups considerations showed a similar pattern among the guidance documents (i.e., $31 \%$ in the use of any EtD framework and $4 \%$ in the use of no framework).

\section{Recommendation-related criteria}

A total of 14 recommendation-related criteria were identified across the guidance documents. Overall, guidance documents that suggested an EtD framework considered a more comprehensive set of criteria than those who did not suggest any framework. Magnitude of desirable and undesirable effects, and certainty of the evidence were the most frequently reported criteria among documents that suggested any framework for guiding the EtD process, with reporting above $80 \%$. In contrast, the use of these three criteria in documents that suggested no framework was $52 \%$. Other criteria such as equity, acceptability, and feasibility were reported in less than half of the documents that guided the EtD process through a systematic framework (any EtD framework), whereas considerably lower rates of use were observed among the documents that did not suggest a framework to guide the EtD process (e., from 0 to $22 \%$ ). Of note, one document reported on the consideration of legal consequences (Domus Medica), and on bioethical considerations (Italian National Center for Clinical Excellence, Quality, and Security, CNEC), respectively.

Among the documents that suggested a framework for guiding the EtD process, those that suggest the GRADE-EtD framework addressed a larger number of criteria compared to those that suggested another framework for the EtD process (see Table 3). Rates of use differed between the two categories. For instance, $95 \%$ of the guidance documents that suggested the GRADE-EtD framework reported the consideration of patients' values relative to $46 \%$ of those that used another EtD framework. A larger difference was observed for equity considerations, $42 \%$ in the GRADE-EtD framework and $11 \%$ in another EtD framework, respectively. Table 2 presents all the recommendation-related criteria reported in the guidance documents. Results from the bivariate analysis are presented in the section below. 
Table 2

Recommendation-related criteria in the EtD process

\begin{tabular}{|c|c|c|c|c|c|}
\hline Criteria & $\begin{array}{l}\text { All guidance } \\
\text { documents } \\
68(100 \%)\end{array}$ & $\begin{array}{l}\text { Any } \\
\text { framework } \\
45 / 68 \\
(66 \%)\end{array}$ & $\begin{array}{l}\text { No } \\
\text { framework } \\
23 / 68 \\
(34 \%)\end{array}$ & $\begin{array}{l}\text { GRADE- } \\
\text { EtD }{ }^{1} \\
19 / 45 \\
(42 \%)\end{array}$ & $\begin{array}{l}\text { Other } \\
\text { framework } \\
26 / 45 \\
(58 \%)\end{array}$ \\
\hline Problem priority & $40(59 \%)$ & $30(67 \%)$ & $10(43 \%)$ & $\begin{array}{l}12 \\
(63 \%)\end{array}$ & $18(69 \%)$ \\
\hline Desirable effects & 49 (72\%) & 37 (82\%) & $12(52 \%)$ & $\begin{array}{l}17 \\
(89 \%)\end{array}$ & $20(77 \%)$ \\
\hline Undesirable effects & $50(73 \%)$ & $38(84 \%)$ & $12(52 \%)$ & $\begin{array}{l}17 \\
(89 \%)\end{array}$ & $21(81 \%)$ \\
\hline $\begin{array}{l}\text { Certainty of the evidence of } \\
\text { effects }\end{array}$ & $50(73 \%)$ & $38(84 \%)$ & $12(52 \%)$ & $\begin{array}{l}18 \\
(95 \%)\end{array}$ & $20(77 \%)$ \\
\hline $\begin{array}{l}\text { Values (outcome } \\
\text { importance) }\end{array}$ & $35(51 \%)$ & $30(67 \%)$ & $5(22 \%)$ & $\begin{array}{l}18 \\
(95 \%)\end{array}$ & $12(46 \%)$ \\
\hline Balance of effects & $39(57 \%)$ & $32(71 \%)$ & $7(30 \%)$ & $\begin{array}{l}17 \\
(89 \%)\end{array}$ & $15(58 \%)$ \\
\hline Resources required & 37 (54\%) & $31(69 \%)$ & $6(26 \%)$ & $\begin{array}{l}18 \\
(95 \%)\end{array}$ & $13(50 \%)$ \\
\hline $\begin{array}{l}\text { Certainty of evidence of } \\
\text { required resources }\end{array}$ & $17(25 \%)$ & $16(36 \%)$ & $1(4 \%)$ & $9(47 \%)$ & $7(27 \%)$ \\
\hline Cost-effectiveness & $36(53 \%)$ & $30(67 \%)$ & $6(26 \%)$ & $\begin{array}{l}16 \\
(84 \%)\end{array}$ & $14(54 \%)$ \\
\hline Equity & $11(16 \%)$ & $11(24 \%)$ & 0 & $8(42 \%)$ & $3(11 \%)$ \\
\hline Acceptability & $19(28 \%)$ & $17(38 \%)$ & $2(9 \%)$ & $9(47 \%)$ & $8(31 \%)$ \\
\hline Feasibility & $23(34 \%)$ & $18(40 \%)$ & $5(22 \%)$ & $9(47 \%)$ & $9(35 \%)$ \\
\hline \multicolumn{6}{|c|}{$\begin{array}{l}{ }^{1} \text { Guidance documents that suggested the GRADE-EtD frameworks for the process of formulating } \\
\text { recommendations. }\end{array}$} \\
\hline م- & ndatis & not & 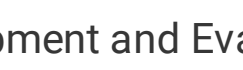 & otion & oach \\
\hline
\end{tabular}


Table 3

Drawing conclusions

\begin{tabular}{|c|c|c|c|c|c|}
\hline Criteria & $\begin{array}{l}\text { All guidance } \\
\text { documents } \\
68(100 \%)\end{array}$ & $\begin{array}{l}\text { Any } \\
\text { framework } \\
45 / 68 \\
(66 \%)\end{array}$ & $\begin{array}{l}\text { No } \\
\text { framework } \\
23 / 68 \\
(34 \%)\end{array}$ & $\begin{array}{l}\text { GRADE- } \\
\text { EtD1 } \\
19 / 45 \\
(42 \%)\end{array}$ & $\begin{array}{l}\text { Other } \\
\text { framework } \\
26 / 45 \\
(58 \%)\end{array}$ \\
\hline $\begin{array}{l}\text { Summary of the judgments } \\
\text { made about the different } \\
\text { criteria considered }\end{array}$ & $28(41 \%)$ & $22(49 \%)$ & $6(26 \%)$ & $9(47 \%)$ & $13(50 \%)$ \\
\hline $\begin{array}{l}\text { Justification of the } \\
\text { recommendation }\end{array}$ & $28(41 \%)$ & $24(53 \%)$ & $4(17 \%)$ & $\begin{array}{l}11 \\
(58 \%)\end{array}$ & $13(50 \%)$ \\
\hline Subgroup considerations & $13(19 \%)$ & $12(27 \%)$ & $1(4 \%)$ & $7(37 \%)$ & $5(19 \%)$ \\
\hline Implementation considerations & $38(56 \%)$ & $31(69 \%)$ & $7(30 \%)$ & $\begin{array}{l}14 \\
(74 \%)\end{array}$ & $17(65 \%)$ \\
\hline $\begin{array}{l}\text { Monitoring and evaluation } \\
\text { considerations }\end{array}$ & $30(44 \%)$ & $25(56 \%)$ & $5(22 \%)$ & $\begin{array}{l}14 \\
(74 \%)\end{array}$ & $11(42 \%)$ \\
\hline Research priorities & $19(28 \%)$ & $18(40 \%)$ & $1(4 \%)$ & $9(47 \%)$ & $9(35 \%)$ \\
\hline
\end{tabular}

Although the wording of some recommendation-related criteria may vary across the documents, which might be explained by the organization preferences, this refers to the same set of criteria described in Table 2. Criteria wording variation is expected for organizations that adapt a framework and process for arriving at recommendations. Thus, some organizations may have addressed the magnitude of the problem before assessing the evidence pertaining the EtD process, and therefore might not report this as an independent criterion in their EtD framework.

To illustrate this better, the GRADE-EtD framework presents a set of additional considerations, so called detailed judgments, that assist the panel when considering the evidence that underlie the main criteria (4). For example, legal consequences, reported by Domus Medica as a specific criterion, would be covered by the GRADE-EtD framework as one of the detailed judgements included under feasibility (i.e., are there important legal or bureaucratic constraints that that make it difficult or impossible to cover the intervention?). The same principle applies to ethical considerations, which is suggested by the Italian Center for Clinical Excellence, Quality and Safety of Care (CNEC), and are addressed as a detailed judgement under acceptability by the GRADE-EtD framework (i.e., are there key stakeholders who would disapprove of the intervention morally, for reasons other than its effects on people's autonomy (such as regarding ethical principles such as no maleficence, beneficence, or justice)?) (4). 


\section{Drawing conclusions as part of the EtD process}

Overall, $41 \%$ of the guidance documents reported a process to summarize the judgments made about the different recommendation-related criteria. This step was more common in the documents that suggested the use of a framework (49\%) compared to documents that did not (26\%). We did not observe major differences on this step between those suggesting the use of the GRADE-EtD framework or another framework ( $47 \%$ vs $50 \%$, respectively). Similarly, the justification of the recommendation's strength and direction was more common in documents that used a framework ( $53 \%$ vs $17 \%$, respectively). This trend was also observed for other steps, such as considerations for relevant subgroups, implementation, monitoring, and evaluation, as well as about the formulation priorities for further research (see Table 3).

\section{Bivariate analysis}

As stated in the methods section, we express the results of the bivariate analysis as the probabilities of addressing each recommendation-related criterion in the different categories of the EtD process (i.e., any framework vs no framework; GRADE-EtD vs other EtD framework; GRADE-EtD vs no framework; other EtD framework vs no framework). The following is a summary of the main findings. We refer the reader to Additional file 4 for a full description of the data.

The use of an EtD framework for guiding the recommendation formulation compared to no framework resulted in higher probability of incorporating both perspectives (OR $2.8 ; 95 \% \mathrm{Cl} 0.6$ to 13.8 ) and subgroup considerations (OR 7.2; $95 \% \mathrm{Cl} 0.9$ to 57.9). Similarly, the documents that incorporated the GRADE-EtD framework were more likely to incorporate these criteria in the recommendation formulation process than those that suggested another EtD framework or no framework, with ORs ranging from 1.4 to 8.4 , respectively.

The probability of using all the recommendation-related criteria identified in this study were higher in documents that suggested the use of any EtD framework relative to no framework, as well as for the documents that suggested the GRADE-EtD framework compared to those that suggested another framework or no framework (see Figure 3).

For instance, guidance documents that suggested the use of any EtD framework were more likely to consider patients' values in the recommendation formulation process compared to those that did not follow any framework (OR $3.1 ; 95 \% \mathrm{Cl} 1$ to 8.9 ). The odds of including patients' values were two-fold greater in documents that suggested the GRADE-EtD framework relative to those that suggested another framework (OR $2 ; 95 \% \mathrm{Cl} 0.8$ to 5.4). The odds ratio increased to 4 when the GRADE-EtD was compared to no framework (OR $4.4 ; 95 \% \mathrm{Cl} 1.4$ to 13.9 ) (see Figure 3).

The guidance documents that suggested any EtD framework were more likely to present evidence on the balance between desirable and undesirable effects when formulating recommendations than documents that did not suggest any framework (OR $2.3 ; 95 \% \mathrm{Cl} 0.9$ to 6.1$)$. The odds were larger for the comparison 
of the documents that suggested the GRADE-EtD framework with those that used no framework (OR 2.9; $95 \% \mathrm{Cl} 1.1$ to 8.6$)$. Similar odds were observed for the criterion related to resources required.

The Odds Ratios (ORs) are based on the comparison of each category of EtD framework relative to the use of no framework. The vertical lines illustrate the $95 \%$ confidence intervals (Cls).

Suggesting the use of an EtD framework was associated with higher odds of including cost-effectiveness considerations when formulating recommendations relative to the use of no framework (OR $2.5 ; 95 \% \mathrm{Cl}$ 0.9 to 7.1). Alike other criteria, the documents that suggested the use of the GRADE-EtD framework were more likely to incorporate cost-effectiveness considerations in the recommendation formulation process than those that suggested another framework (OR 1.6; $95 \% \mathrm{Cl} 0.6$ to 3.9 ) or no framework (OR $3.2 ; 95 \% \mathrm{Cl}$ 1.1 to 9.8 ) (Figure 3).

Of note, the odds of including recommendation-related criteria such as equity, acceptability, and feasibility followed the same pattern. That is, the use of any EtD framework and the GRADE-EtD framework resulted in higher probability of including those criteria when formulating recommendations relative to the use of no framework or another EtD framework.

Finally, and in line with the associations observed for the recommendation-related criteria, the documents that suggested both the use of any framework or the GRADE-EtD framework were more likely to provide a justification of the judgements made, implementation considerations, as well as monitoring and evaluation considerations than the documents that suggested no framework or another framework. Additional file 4 presents further details.

\section{Discussion}

\section{Main findings}

Our study presents a complete and systematic evaluation of the frameworks and processes suggested when moving from evidence to recommendations in guideline development. We documented the diversity of both frameworks and processes in this area. GRADE emerged as the most common approach for grading both the certainty of the evidence and the strength of recommendations. Our analyses revealed that only slightly more than half of the organizations presented a structured process for formulating recommendations, in their guidance documents. However, the analyses also showed that there are gaps in important aspects like the sharing of preliminary material or judgements through the recommendation formulation process.

Fourteen recommendation-related criteria were identified in the guidance documents. The use of an EtD framework was associated with higher probabilities of addressing a comprehensive set of recommendation-related criteria compared to the use of no framework, especially for criteria like patients' values and preferences, equity, and acceptability. Similarly, the use of the GRADE-EtD framework was associated with higher probabilities of including those criteria when compared to other EtD frameworks 
or no framework. However, caution is advised when drawing inferences from the analysis due to the small number of guidance documents included in our analysis.

\section{Our results in the context of previous research}

Previous research suggests the GRADE-EtD framework is a widely used tool for assisting comprehensive and transparent evidence-informed decision making $(12 ; 13 ; 24 ; 25)$. Our findings confirm this notion, since the methods developed by the GRADE Working Group were the most used approach. For example, a case study conducted by members of the GRADE working group (12) showed that the GRADE-EtD coverage framework had been fully accepted by some stakeholders in the United States (24). Similarly, a more recent real-time assessment of the use of the GRADE-EtD framework in guideline panels concluded that it was essential to structure panel meetings and ensure the consideration of all relevant criteria (17). In that study, formal GRADE-EtD's criteria guided $94 \%$ of panel discussions, whereas other external criteria guided the remaining $6 \%$ of the discussions (e.g., clinical experience, political environment, and legal implications). Nevertheless, both the extent to which the criteria contained in the GRADE-EtD framework restraint discussions among panelists, and the need for additional criteria remain unexplored (17).

Despite the diversity of both, frameworks and processes in this area, there is still ongoing debate and research on the adequacy and validity of available frameworks for different types of decisions (26) and organizations (10). Recently, different authors have suggested the need of including additional criteria in the GRADE EtD framework. For example, burden of treatment (5) or human rights and sociocultural acceptability, equality and non-discrimination, societal implications, and health system considerations by other authors (10). The need for tailoring for different types of organizations or goals was considered in the GRADE-EtD frameworks in the original publications. For example, ethical considerations are considered under acceptability in the GRADE-EtD frameworks (see detailed judgments). Therefore, an organization might consider treating ethical considerations as a separate criterion, rather than as a detailed judgment under acceptability (4).

\section{Limitations and strengths}

Our study had some limitations. We do not rule out having excluded documents that organizations may have published in other languages. Nevertheless, we do not anticipate missing relevant information, as our searches covered a representative number of guidance documents derived from the most relevant organizations in the field.

Regarding the strengths, we followed methodological standards for the conduct and reporting of evidence synthesis studies, such as systematic search of the literature, double screening process, and a priori piloting of the data extraction procedures. Data were analyzed through iterative consensus between researchers. The project team was strategically positioned to carry out this study, as the researchers are experienced in working with guideline panels and are experts in evidence synthesis methods and the guideline development process.

\section{Implications for practice and research}


Our findings provide a systematic analysis of different criteria contained in the frameworks proposed for the recommendation formulation process among guidance documents for guideline development. It is important to remark that the information extracted from the guidance documents was reliant on how complete the information about each criterion was presented in the documents, and so was our analysis. As an example of this, stating the organization used the GRADE-EtD framework did not equal the inclusion of all the recommendation-related criteria proposed by the framework; this was also true for other frameworks proposed by the organizations. Therefore, all organizations should follow a more systematic and explicit methodology for formulating recommendations and ensure a complete reporting in their guidance documents.

We systematically documented the use of EtD frameworks in guidance documents; however, the extent to which the mention of the different criteria in the guidance documents translates into a real use in published guidelines is still unknown. Besides, since both guideline developers and panel members have expressed favorable experiences with the use of EtD frameworks $(12 ; 17 ; 24)$, further qualitative research exploring the use of the frameworks among panelists and methodologist is also warranted. Our findings could also foster further research on the need of modifications of available frameworks.

\section{Conclusion}

The use of systematic and structured processes for moving from evidence to decisions is still limited among international organizations devoted to guideline development. The use of EtD frameworks facilitates the inclusion of relevant recommendation criteria. Among the structured frameworks, the GRADE-EtD framework is the most widely used approach, offering the most comprehensive perspective for evidence-informed decision-making processes. More complete and detailed reporting in the guidance documents is warranted.

\section{List Of Abbreviations}

EtD; Evidence-to-Decision

GDG; Guideline Development Group

GIN; Guideline International Network

GPS; Global Priority Setting

GRADE; The Grading of Recommendations Assessment, Development and Evaluation approach

WHO; World Health Organization

\section{Declarations}

\section{Funding}


No funding is received for this research project.

\section{Acknowledgements}

We thank Andrea Juliana Sanabria (AJS) and Anna Selva (AS) for their support and assistance with the literature searches. Besides, we thank the Norwegian Institute of Public Health for their support with the publication fee for this study.

\section{Availability of data and materials}

Not applicable. Datasets are available from the correspondence author upon request.

\section{Author's contributions}

JFME and PAC conceptualized the idea for this study. All authors contributed to the development of the selection criteria, and the development of the data extraction form. AJS, AS, and JFME conducted the literature searches. HS provided the updated versions of some guidance documents. JFME, PAC, and JB conducted the initial pilot of the data extraction form, and the rest of the team tested it for standardization procedures. JFME, JB, JJYN, TPP, LP, MMB, DS, MS, JZ, CMG, YZ, NCG, and PAC extracted data from the guidance documents. HS, SF, PAC, and JFME drafted the manuscript. All authors read, provided feedback, and approved the final manuscript.

\section{Ethics approval and consent to participate}

Not applicable.

\section{Consent for publication}

Not applicable

\section{Competing interests}

The authors declare that they have no competing interests. Jose Meneses-Echavez is a doctoral candidate in Public Health and Methodology of Biomedical Research, at the Department of Paediatrics, Obstetrics, Gynaecology and Preventive Medicine at Universitat Autonoma de Barcelona, Spain. Pablo Alonso-Coello, Signe Flottorp, and Holger Schünemann were involved in the development of the GRADEEtD framework.

\section{References}

1. Alonso-Coello P, Martinez Garcia L, Carrasco JM, Sola I, Qureshi S, Burgers JS. The updating of clinical practice guidelines: insights from an international survey. Implementation science: IS. 2011;6:107. 
2. The National Academies Press. editor. IOM (Institute of Medicine). Clinical Practice Guidelines We Can Trust. Washington, DC: 2011.

3. Schunemann HJ, Wiercioch W, Etxeandia I, Falavigna M, Santesso N, Mustafa R, et al. Guidelines 2.0: systematic development of a comprehensive checklist for a successful guideline enterprise. CMAJ: Canadian Medical Association journal = journal de l'Association medicale canadienne. 2014;186(3):E123-42.

4. Alonso-Coello P, Schünemann HJ, Moberg J, Brignardello-Petersen R, AkI EA, Davoli M, et al. GRADE Evidence to Decision (EtD) frameworks: a systematic and transparent approach to making well informed healthcare choices. 1: Introduction. BMJ 2016;353:i2016.

5. Dobler CC, Harb N, Maguire CA, Armour CL, Coleman C, Murad MH. Treatment burden should be included in clinical practice guidelines. BMJ. 2018;363:k4065.

6. Goetghebeur MM, Wagner M, Khoury H, Levitt RJ, Erickson LJ, Rindress D. Evidence and Value: Impact on DEcisionMaking--the EVIDEM framework and potential applications. BMC Health Serv Res. 2008;8:270.

7. World Health Organization. WHO handbook for guideline development. 2nd ed. Geneva: World Health Organization; 2014.

8. Tannahill A. Beyond evidence-to ethics: a decision-making framework for health promotion, public health and health improvement. Health Promot Int. 2008;23(4):380-90.

9. Norheim OF, Baltussen R, Johri M, Chisholm D, Nord E, Brock D, et al. Guidance on priority setting in health care (GPS-Health): the inclusion of equity criteria not captured by cost-effectiveness analysis. Cost effectiveness and resource allocation: C/E 2014;12:18.

10. Rehfuess EA, Stratil JM, Scheel IB, Portela A, Norris SL, Baltussen R. The WHO-INTEGRATE evidence to decision framework version 1.0: integrating WHO norms and values and a complexity perspective. BMJ Global Health. 2019;4(Suppl 1):e000844.

11. Morgan RL, Thayer KA, Bero L, Bruce N, Falck-Ytter Y, Ghersi D, et al. GRADE: Assessing the quality of evidence in environmental and occupational health. Environ Int. 2016;92-93:611-6.

12. Neumann I, Brignardello-Petersen R, Wiercioch W, Carrasco-Labra A, Cuello C, Akl E, et al. The GRADE evidence-to-decision framework: a report of its testing and application in 15 international guideline panels. Implementation science: IS. 2016;11:93.

13. Rosenbaum SE, Moberg J, Glenton C, Schünemann HJ, Lewin S, AkI E, et al. Developing Evidence to Decision Frameworks and an Interactive Evidence to Decision Tool for Making and Using Decisions and Recommendations in Health Care. Global Challenges. 2018;2(9):1700081.

14. American College of Chest Physicians (CHEST). Guideline Development[cited March, 2021]. Available from: https://www.chestnet.org/Guidelines-and-Resources/About-CHEST-Guidelines/GuidelineDevelopment.

15. Canadian Task Force on Preventive Health Care. Canadian Task Force on Preventive Health Care Procedure Manual. 2014. Available from: https://canadiantaskforce.ca/methods/. 
16. Centro Nazionale per l'Eccellenza Clinica la Qualità e la Sicurezza delle Cure. Manuale metodologico per la produzione di linee guida di pratica clinica. 2019. Available from: https://snlg.iss.it/wpcontent/uploads/2019/04/MM_v1.3.2_apr_2019.pdf.

17. Li SA, Alexander PE, Reljic T, Cuker A, Nieuwlaat R, Wiercioch W, et al. Evidence to Decision framework provides a structured "roadmap" for making GRADE guidelines recommendations. J Clin Epidemiol. 2018;104:103-12.

18. Higgins JPTTJ, Chandler J, Cumpston M, Li T, Page MJ, Welch VA, editors. Cochrane Handbook for Systematic Reviews of Interventions version 6.2 (updated February 2021): Cochrane [cited]. Available from: .

19. Meneses-Echavez JF, Bidonde J, Yepes-Nuñez JJ, Storman D, Puljak L, Zając J, et al. Moving from evidence to decisions when making recommendations in healthcare: a protocol of an analysis of clinical practice guidelines guidance documents March 2020: Open Science Framework [cited]. Available from: osf.io/uzjmb.

20. Vernooij RW, Sanabria AJ, Sola I, Alonso-Coello P, Martinez Garcia L. Guidance for updating clinical practice guidelines: a systematic review of methodological handbooks. Implementation science: IS. 2014;9:3.

21. Selva A, Sanabria AJ, Pequeno S, Zhang Y, Sola I, Pardo-Hernandez H, et al. Incorporating patients' views in guideline development: a systematic review of guidance documents. J Clin Epidemiol. 2017;88:102-12.

22. Schunemann HJ, Oxman AD, Akl EA, Brozek JL, Montori VM, Heffner J, et al. Moving from evidence to developing recommendations in guidelines: article 11 in Integrating and coordinating efforts in COPD guideline development. An official ATS/ERS workshop report. Proceedings of the American Thoracic Society 2012;9(5):282-92.

23. R Core Team. R: A language and environment for statistical computing. I: R Foundation for Statistical Computing, editor. Vienna, Austria2017.

24. Dahm P, Oxman AD, Djulbegovic B, Guyatt GH, Murad MH, Amato L, et al. Stakeholders apply the GRADE evidence-to-decision framework to facilitate coverage decisions. J Clin Epidemiol. 2017;86:129-39.

25. Meneses-Echavez JF, Rosenbaum S, Rada G, Flottorp S, Moberg J, Alonso-Coello P. Users' experiences with an interactive Evidence to Decision (iEtD) framework: a qualitative analysis. BMC Med Inf Decis Mak. 2021;21(1):169.

26. Morgan RL, Kelley L, Guyatt GH, Johnson A, Lavis JN. Decision-making frameworks and considerations for informing coverage decisions for healthcare interventions: a critical interpretive synthesis. J Clin Epidemiol. 2018;94:143-50.

\section{Figures}




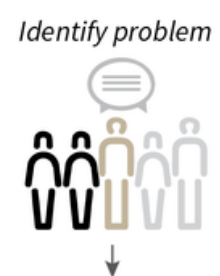

Formulate question

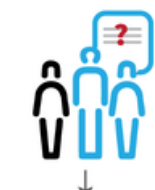

Populate EtD framework

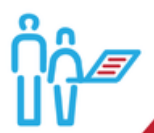

Make evidence-informed judgments

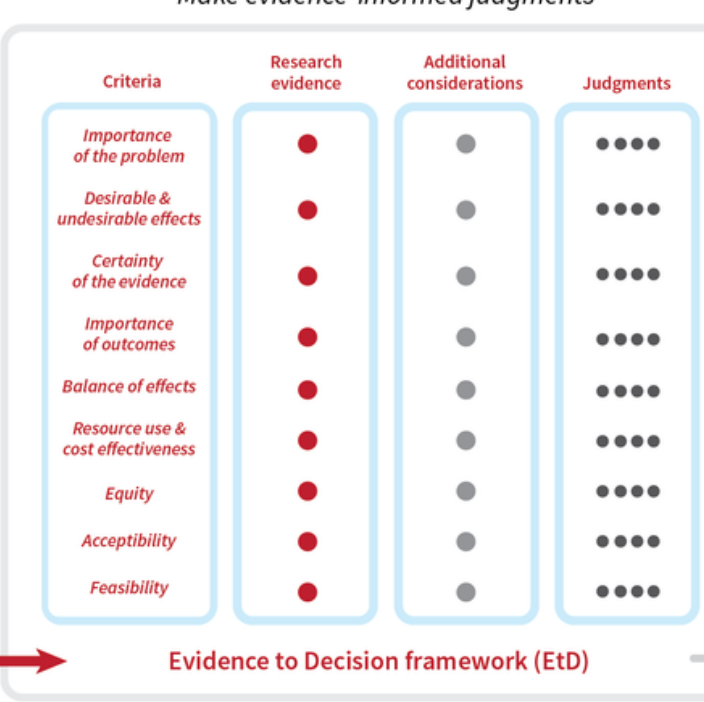

Recommend

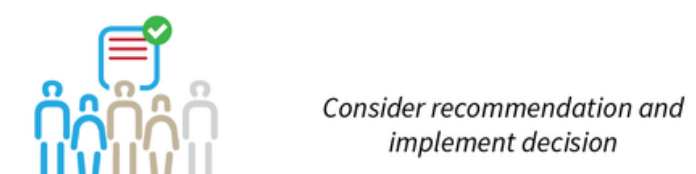

\section{Figure 1}

GRADE-EtD frameworks workflow

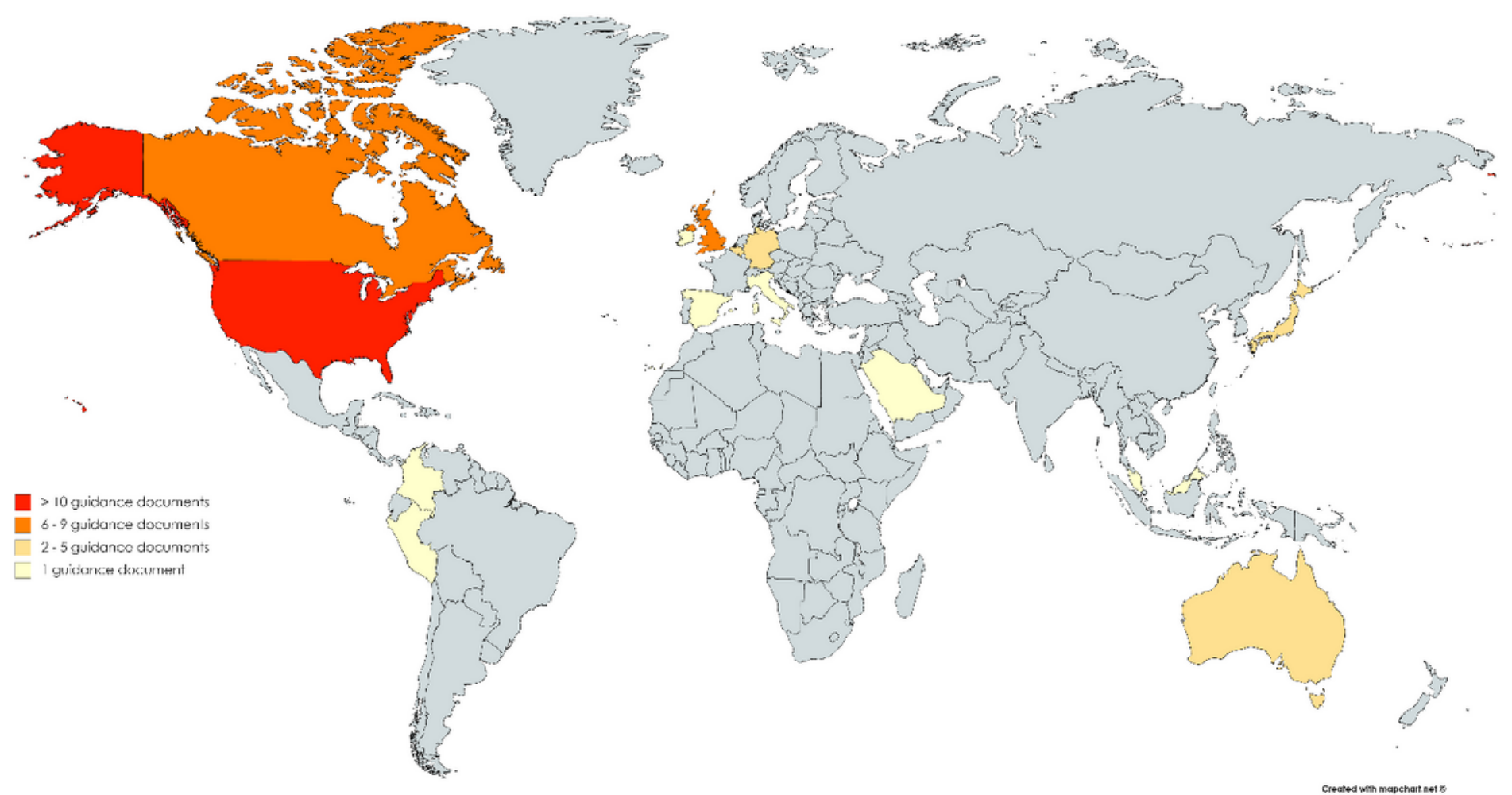

Figure 2 


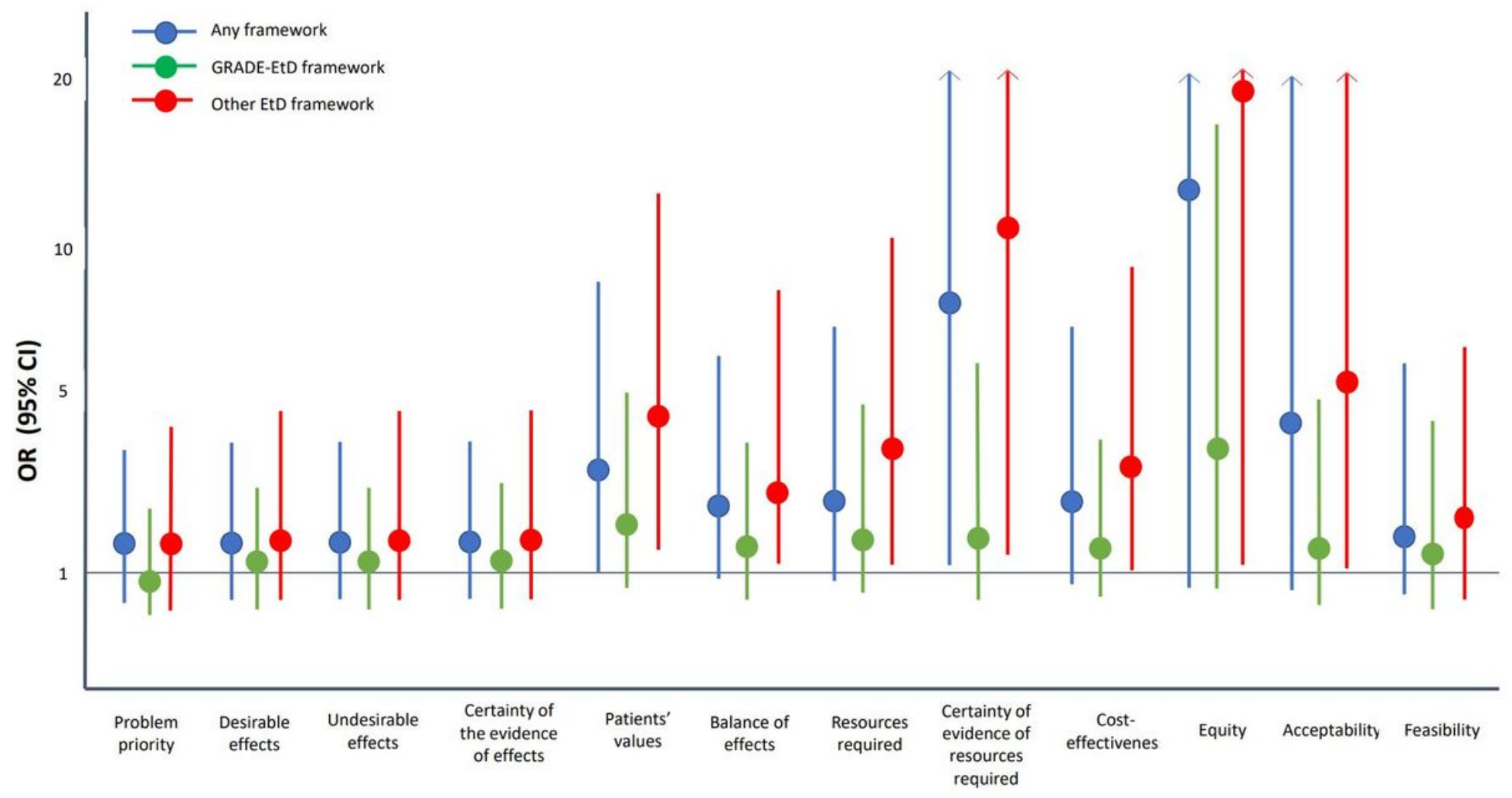

Figure 3

Bivariate analysis for the recommendation-related criteria in the guidance documents

\section{Supplementary Files}

This is a list of supplementary files associated with this preprint. Click to download.

- Additionalfile1.docx

- Additionalfile2.docx

- Additionalfile3.docx

- Additionalfile4.docx

- PRISMA2020checklist.docx 